\title{
Regeneración Ósea en Defectos Críticos Tratados con Matriz Ósea Bovina Inorgánica Aplicada con dos Diferentes Vehículos
}

\author{
Bone Regeneration in Critical Defect Treated with Bovine \\ Inorganic Bone Matrix with two Different Carriers
}

\author{
Henrique Duque Netto*; Sergio Olate*,***; Leandro Klüppel ${ }^{* * * * ;}$ Maria das Graças Alfonso de Miranda Chaves*; \\ Ivone de Oliveira Salgado*; Bélgica Vásquez ${ }^{* * * * *}$ \& Jose Albergaria-Barbosa ${ }^{* * * * * *}$
}

DUQUE NETTO, H.; OLATE, S.; KLÜPPEL, L.; MIRANDA CHAVES, M. G. A.; SALGADO, I. O.; VÁSQUEZ, B. \& ALBERGARIA-BARBOSA, J. Regeneración ósea en defectos críticos tratados con matriz ósea bovina inorgánica aplicada con dos diferentes vehículos. Int. J. Morphol., 31(2):367-372, 2013.

RESUMEN: Múltiples materiales se han utilizado en la reconstrucción ósea; la matriz ósea bovina ha sido ampliamente estudiada y aun existen investigaciones que identifican su capacidad de contribuir en la formación ósea. El objetivo de esta investigación fue establecer la reparación ósea en defectos críticos de calotas de perros rellenados con matriz ósea bovina inorgánica transportada con carboxi-metil-celulosa. Se diseñó un estudio experimental en 6 perros donde se realizaron 3 defectos de $8 \mathrm{~mm}$ de diámetro en los parietales de cada animal; el defecto fue rellenado aleatoriamente con hueso autógeno particulado (grupo I), matriz ósea bovina inorgánica con suero fisiológico al $0,9 \%$ (grupo II) y matriz ósea bovina con carboxi-metil-celulosa (grupo III). Se realizó el sacrificio de los animales a la tercera y sexta semana momento en el que se realizaron los estudios radiográficos de los defectos tratados y los estudios histológicos de rutina con tinciones de hematoxilina-eosina. No se observó procesos infecciosos vinculados a los materiales injertados. En el grupo del hueso autógeno se observó una adecuada aposición ósea en la tercera y sexta semana junto a una relación correcta con la imagen radiográfica; en el grupo II se observó etapas de formación ósea y permanencia de los fragmentos de hueso injertado; en el grupo III se observó infiltrado inflamatorio en los dos periodos de sacrificio, abundante tejido conectivo y bajo nivel de formación ósea. Las radiografías de los grupos II y III mostraron signos de formación ósea que en la histología eran sólo remanentes del material injertado. Se concluye que la matriz ósea bovina inorgánica es compatible con el tejido óseo y que puede contribuir a la formación ósea aunque el empleo de la carboxi-metil-celulosa, como vehículo, podría obstaculizar la regeneración ósea.

PALABRAS CLAVE: Hueso; Hueso bovino; Injerto óseo.

\section{INTRODUCCIÓN}

La regeneración ósea con diferentes materiales ha sido el objetivo de múltiples investigaciones. La disminución de la morbilidad quirúrgica, la disminución del tiempo quirúrgico y los costos de hospitalización han fundamentado gran parte de esta investigación (Grageda, 2004). Aunque el injerto óseo autógeno es el patrón oro en las diferentes investigaciones (Moore et al., 2001., Olate et al., 2007) diversos materiales y métodos de instalación continúan siendo experimentados para intentar optimizar los resultados (Olate et al., 2007).
La matriz ósea bovina es un material de amplia investigación con fuerte respaldo en la literatura internacional (Degidi et al., 2005). Su empleo ha sido llevado a cirugías reconstructivas extensas así como a procedimientos menores utilizándose también en combinación con otros biomateriales (Serra e Silva et al., 2006). Hollinger et al., (1990) inicialmente señalaron que este material presenta una propiedad osteoconductiva innegable, lo que le confiere al material ciertas capacidades para ser instalado en algunos defectos óseos; esto se logró, sin embargo, luego

Universidad Federal de Juiz de Fora, Brasil.

** Universidad de La Frontera, Chile.

*** Centro de Investigación en Ciencias de la Salud, Universidad Autónoma de Chile, Chile.

***** Universidad Estadual de Ponta Grossa, Brasil.

****** Universidad de Tarapacá, Chile.

******** Universidad Estadual de Campinas, Brasil.

Proyecto DIUFRO Nº DI12-0015, Universidad de La Frontera, Temuco, Chile. 
de eliminar reacciones auto inmunes y reacciones antígenoanticuerpo que en los primeros momentos se observaron (Basle et al., 1998).

Una vez reconocida la capacidad de contribuir en la formación ósea, la morfología y la forma de instalación del biomaterial es un factor fundamental (Boyne et al., 2005). Ya se ha determinado que el tamaño de la partícula es importante en la regeneración ósea (partículas de $0,5 \mathrm{~mm}^{3}$ a $2 \mathrm{~mm}^{3}$ presentan mayor formación ósea) (Pallesen et al., 2002), por lo tanto, la metodología de transporte como bloque, pasta, partícula, gránulos, entre otros también tiene su papel en la aplicación y el éxito del material. En este sentido, Vaance et al. (2004) estudiaron la formación ósea en defectos tratados por una pasta compuesta de carboxi-metil-celulosa y sulfato de calcio combinado con hueso aloplástico desmineralizado, demostrando que el volumen óseo formado era menor al grupo control pero que la calidad y vascularización ósea eran de mejor nivel en el grupo test. Otras investigaciones también han presentado a la carboxi-metil-celulosa como un elemento que favorecería la instalación del biomaterial ya que al ser aplicado como una pasta, favorece la manipulación y adaptación (Santa-Comba et al., 2000).

El objetivo de esta investigación fue comparar la formación ósea en defectos críticos rellenados con hueso bovino inorgánico en partículas aplicado con suero fisiológico o carboxi-metil-celulosa, utilizando el hueso autógeno particulado como grupo control.

\section{MATERIAL Y MÉTODO}

Esta investigación fue aprobada por el comité de ética en experimentación animal de la Universidad Estadual de Campinas con el protocolo numero 1343-1. Se desarrolló una investigación en un modelo animal canino (6 perros adultos de $15 \mathrm{~kg}$ aproximadamente) creando defectos críticos de $8 \mathrm{~mm}$ de diámetro en la calota de los animales.

Procedimiento quirúrgico. Los animales fueron sedados con clorhidrato de ketamina $(0,15 \mathrm{ml} / \mathrm{kg})$ y pentobarbital sódico $(30 \mathrm{mg} / \mathrm{kg})$ además de la aplicación de antibióticos (penicilina benzatina $0,1 \mathrm{ml} / \mathrm{kg}$ ), analgésicos y corticoides (dexametasona $0,5 \mathrm{mg} / \mathrm{kg}$ ).

Posteriormente se realizó la tricotomía craneana junto a las técnicas de asepsia y antisepsia estandar. Se realizó una incisión lineal de 8 a $10 \mathrm{~cm}$ a nivel sagital y el posterior descolamiento de los huesos parietales. Una vez expuesto el tejido óseo, se fabricaron 3 defectos de $8 \mathrm{~mm}$ de diámetro, bicorticales, con una trefina de $8 \mathrm{~mm}$ de diámetro montada en un motor de baja velocidad (20.000rpm aproximadamente); la trefina llegó hasta el límite de la cortical con la dura madre; se realizó una gentil manipulación con descoladores que permitieran desplazar el fragmento sin dañar la dura madre. Una vez terminada esta fase se procedió a rellenar los defectos seleccionando con un material distinto en cada defecto, siendo las opciones el mismo hueso retirado que fue particulado con tamaños de entre 750 y 1000 mm (Grupo I), matriz ósea bovina inorgánica aplicada con suero fisiológico con tamaños de entre 450 a 749 mm (Grupo II) y la misma matriz ósea bovina inorgánica junto a carboxi-metil-celulosa como vehículo lo que permitía su inserción con textura de pasta (Consulmat@ Industria e Comercio Ltda.) (Grupo III).

Posteriormente los tejidos blandos fueron reposicionados, el periostio cubrió completamente los defectos y se realizo la sutura por planos utilizando vycril 3-0 (polyglactin 910) e hilo de nylon 4-0 en el tejido cutaneo. Se ejecutaron dos periodos de sacrificio con tres animales en cada periodo; estos periodos fueron a la tercera semana y a la sexta semana.

Obtención de muestras de análisis. Una vez sacrificados los animales, se obtuvo las muestras óseas utilizando fresas de corte en motor de alta rotación (400.000 rpm) respetando al menos $1 \mathrm{~cm}$ de margen de seguridad para seccionar la muestra; en la misma etapa de obtención de la muestra se realizó la toma radiográfica utilizando películas rápidas y un sistema de imagen calibrado en $60 \mathrm{KV}, 10 \mathrm{~mA}$ y 0,25 segundos de exposición. La etapa de revelado tomo 7 minutos y se obtuvo en maquina de revelado automática; las imágenes fueron estudiadas y descritas por un radiólogo maxilofacial con experiencia en el área, el cual desconocía el origen de la imagen analizada.

Las muestras óseas fueron procesadas y obtenidas laminas de $6 \mathrm{~mm}$ de ancho, obteniendo 5 laminas por cada defecto óseo estudiado; se utilizaron técnicas histológicas de rutina con la tinción hematoxilina-eosina.

\section{RESULTADOS}

\section{Análisis Radiográfico}

1. Hueso autógeno. La imagen obtenida a la tercera semana mostró una cavidad bien delimitada y complemente rellena, siendo posible observar áreas radiopacas en su interior. A las seis semanas la periferia del defecto fue menos delimitada y se observó áreas donde se confunde el punto de finalización del defecto original (Fig. 1). 

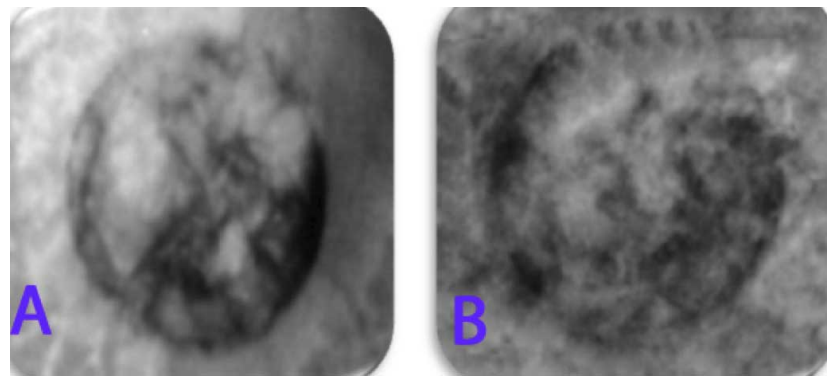

Fig. 1. Imagen radiográfica del defecto creado rellenado con hueso autógeno particulado en la etapa de 3 semanas (A) y 6 semanas (B)

\section{Matriz ósea bovina inorgánica asociada a suero fisio-} lógico $(\mathbf{0 , 9 \%})$. La imagen presentada a la tercera semana permitió observar claramente los límites del defecto; se observa un área radiolúcida bien definida. A la sexta semana, se observó algunos puntos radiopacos centrales y en algunos puntos de la periferia, aunque se observa con claridad todos los limites del defecto óseo (Fig. 2).
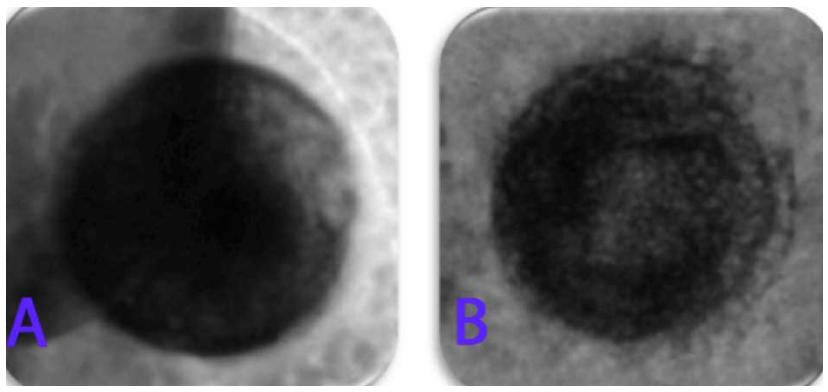

Fig. 2. Imagen radiográfica del defecto creado rellenado con matriz ósea bovina inorgánica en vehículo de suero fisiológico al 0,9\% en la etapa de 3 semanas (A) y 6 semanas (B)
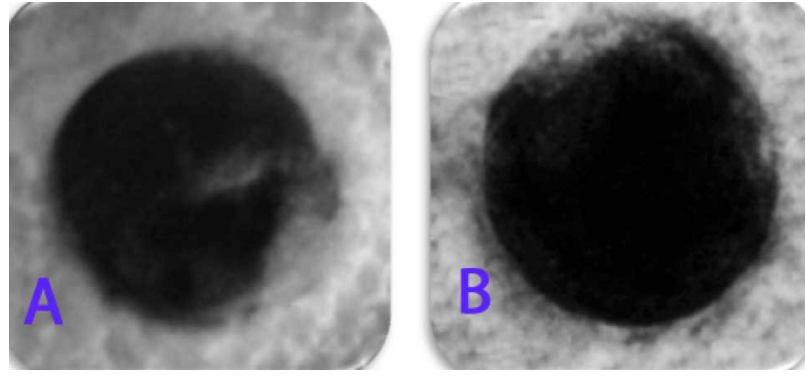

Fig. 3. Imagen radiográfica del defecto creado rellenado con matriz ósea bovina inorgánica en vehículo de suero fisiológico al $0,9 \%$ en la etapa de 3 semanas (A) y 6 semanas (B)

\section{Matriz ósea bovina inorgánica asociada a carboxi-} metil-celulosa. La imagen de la tercera semana muestra bien definido el defecto óseo; no se observó signos imagenológicos de reparación ósea. En la sexta semana se mantiene el área radiolúcida sin alteraciones, observándose claramente la diferencia entre el área de tejido óseo y el área con el defecto óseo (Fig. 3).

\section{Análisis Histológico}

1. Hueso autógeno. A las tres semanas se observó una cavidad que presenta diferencias entre la periferia del defecto y el área central en fase de remodelación ósea; se caracterizó un procedo de formación ósea centrípeta con interposición de tejido conectivo en contacto con el trabeculado óseo junto a áreas de revascularización; en algunas áreas se observo un discreto infiltrado inflamatorio. A la sexta semana se observó un elevado nivel de regeneración ósea con trabeculado óseo y algunas áreas de tejido conectivo; se observaron algunas partículas del injerto óseo utilizado en ausencia de infiltrado inflamatorio; los limites del defecto prácticamente están ausentes en las imágenes estudiadas (Fig. 4).
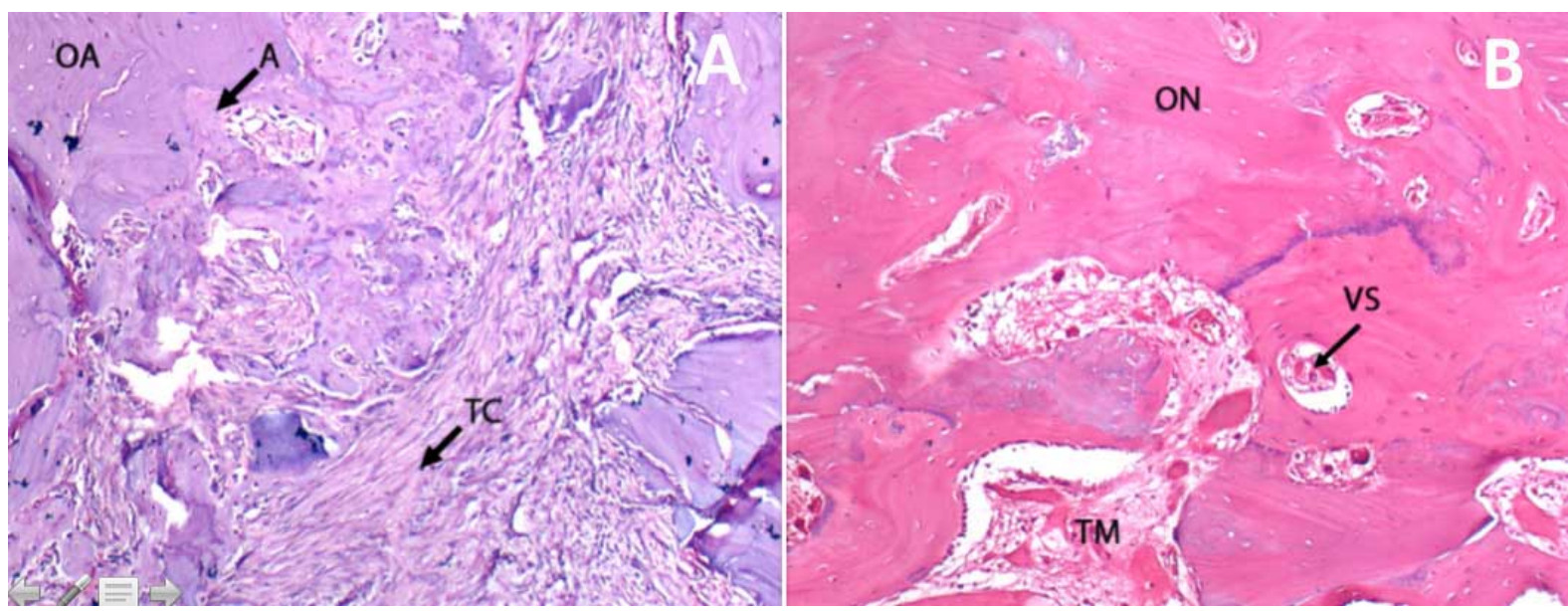

Fig. 4. Imagen microscópica (HE) del defecto con relleno de injerto óseo particulado autógeno (grupo I) en 20X. A: aposición ósea, OA: injerto óseo autógeno, TC: tejido conectivo, ON: tejido óseo neoformado, TM: tejido óseo medular, VS: vaso sanguíneo. A) Condición a la tercera semana y B) Condición a la sexta semana. 
2. Matriz ósea bovina inorgánica asociada a suero fisiológico $(\mathbf{0 , 9 \%})$. La imagen histológica obtenida a la tercera semana reveló la presencia de tejido conectivo abarcando casi la totalidad del defecto creado; se observo limitada formación ósea; al centro de la cavidad se encontró particular óseas remanentes del material injertado; también se observó un discreto infiltrado inflamatorio mayor al encontrado en el grupo de hueso autógeno. A la sexta semana se observan incipientes inicios de vascularización con áreas de necrosis dentro del defecto creado; algunas partículas se encontraban en fase de reabsorción, rodeadas en su mayoría por tejido conectivo; también se observó la permanencia de algún infiltrado inflamatorio rodeando algunas partículas del biomaterial utilizado. Aun se observaba la diferencia entre el tejido óseo preexistente y el área del defecto rellenado con el material (Fig. 5).

\section{Matriz ósea bovina inorgánica asociada a carboxi-} metil-celulosa. En los defectos se observó claramente el limite con el tejido óseo preexistente; en el interior del defecto la gran mayoría del tejido era conectivo en conjunto con el material de relleno; se observaron áreas de remodelación ósea en la periferia del defecto; encontrando también una importante presencia de proceso inflamatorio, mayor al encontrado en los otros grupos. En la sexta semana de postoperatorio se observó la persistencia de parte importante del tejido conectivo con los márgenes de la cavidad formada claramente definidos; se observó permanencia del proceso inflamatorio con limitada formación ósea (Fig. 6).

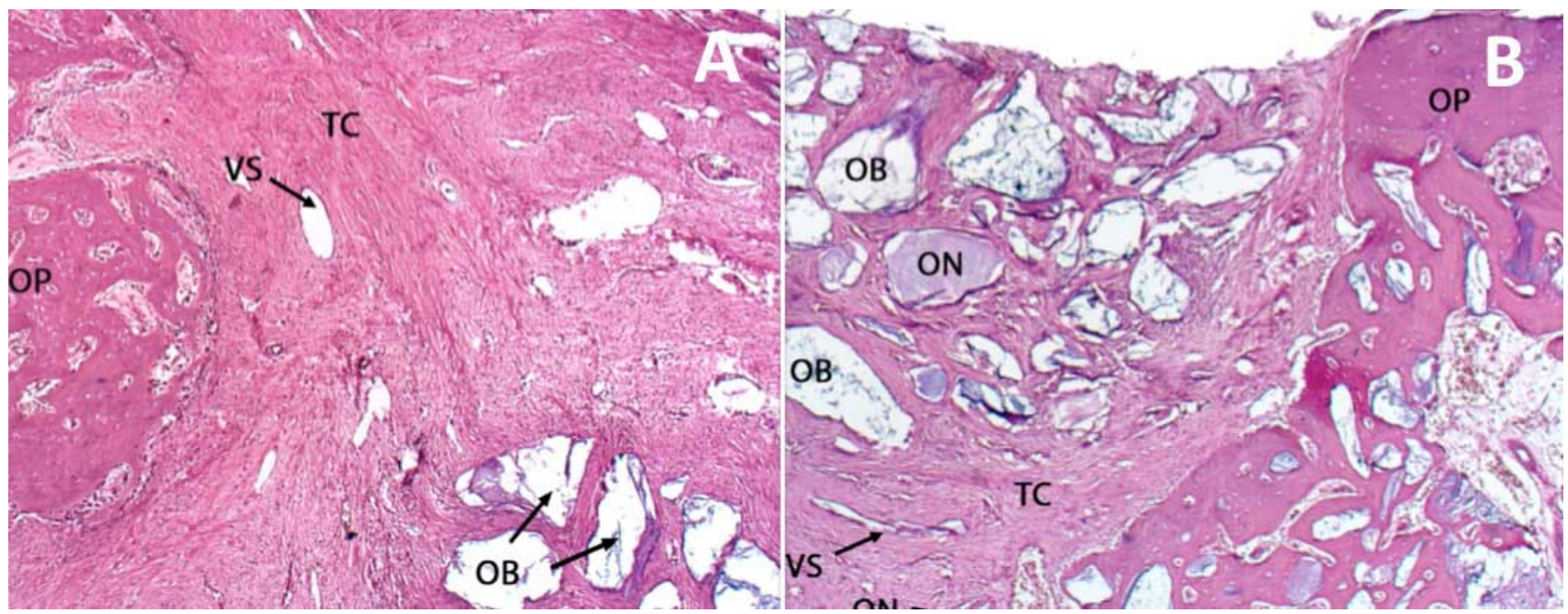

Fig. 5. Imagen microscópica (HE) del defecto con relleno de matriz ósea bovina con vehículo de suero físiológico al 0,9\% (grupo II) en 10X. OP: hueso preexistente, OB: matriz ósea bovina, TC: tejido conectivo, VS: vaso sanguíneo, ON: tejido óseo neoformado. A) Condición a la tercera semana y B) Condición a la sexta semana.

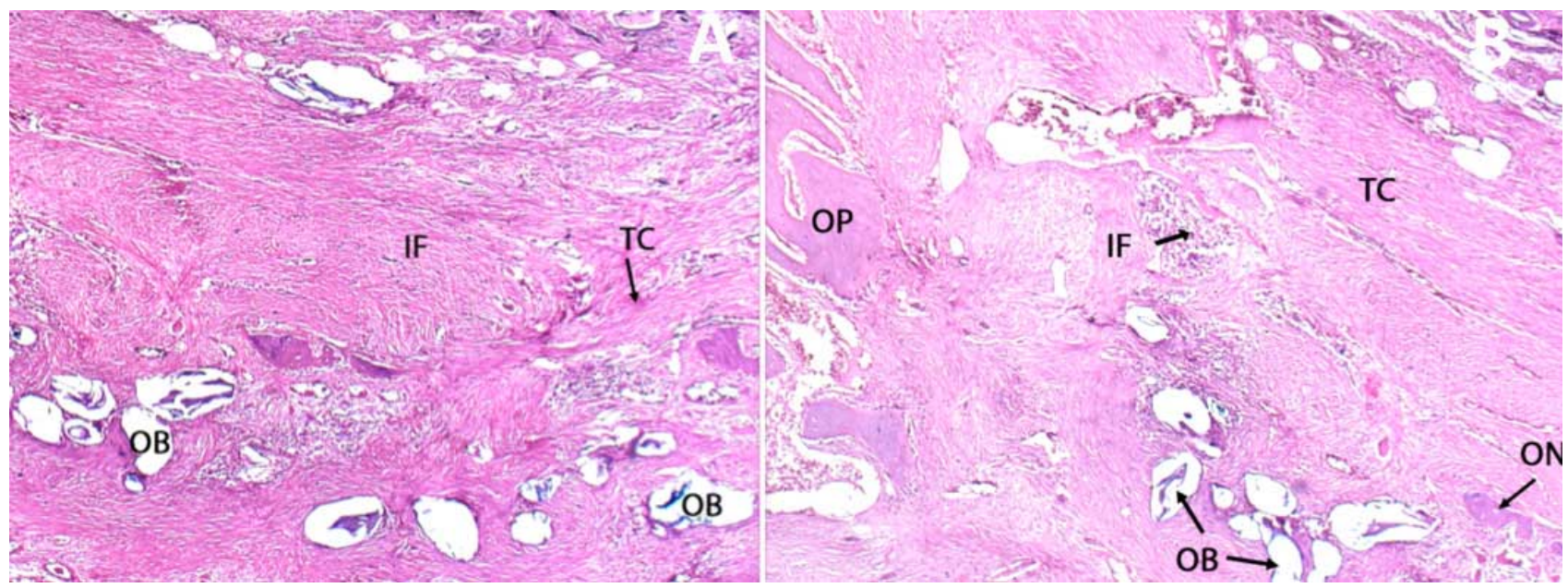

Fig. 6. Imagen microscópica (HE) del defecto con relleno de matriz ósea bovina con vehículo de carboxi-metil-celulosa (grupo III) en 10X. IF: infiltrado inflamatorio, OB: matriz ósea bovina, TC: tejido conectivo, OP: hueso preexistente, ON: tejido óseo neoformado. A) Condición a la tercera semana y B) Condición a la sexta semana. 


\section{DISCUSIÓN}

Los resultados observados en esta investigación muestran que el hueso autógeno continua siendo el de mejor respuesta en la neo formación ósea y también el de más rápida regeneración al ser comparado con el hueso de origen bovino. El uso de la matriz bovina inorgánica es ampliamente aceptada en la literatura (Pallesen et al., Degidi et al.,) siendo utilizada en diferentes condiciones quirúrgicas.

Los materiales testados confirmaron ser biotolerados, propiedad fundamental en los materiales de implantación (Basle et al., Haas et al., 2002). Investigaciones previas (Chaves Netto et al., 2009) mostraron que defectos mayores que $8 \mathrm{~mm}$ en calotas de perros podrían ser considerados defectos críticos, lo cual exige del material implantado la necesidad de estimular la formación ósea; los resultados demostraron que en los grupos de uso de matriz bovina existió un limitado nivel de formación ósea, donde el vehículo a base de suero fisiológico mostró mayores niveles de regeneración ósea al ser comparado con el vehículo de carboxi-metil-celulosa. Siendo el mismo material bovino de relleno en ambos casos, es posible especular sobre el rol del vehículo en la incorporación del injerto óseo, observándose en algunas etapas de esta investigación que la carboxi-metil-celulosa pudo obstaculizar la respuesta ósea más que ayudar a la incorporación del injerto.

Aún existe incertidumbre en como los vehículos podrían interferir en la reparación ósea (Boyne et al., Santa-Comba et al.,); la carboxi-metil-celulosa tiene un alto poder aglutinante entregando propiedades pastosas al material lo cual puede ser muy favorable a la manipulación clínica pero, a la luz de estos resultados, limitó la formación ósea. A diferencia de lo reportado por Santa-Comba et al., observamos una pobre respuesta ósea cuando se utilizo carboxi-metil-celulosa como vehículo de implantación, aumentando la cantidad de tejido conectivo en el defecto con la consecuente disminución de la formación ósea (Haas et al.).

En las imágenes histológicas de la tercera y sexta semana se observó que rodeando las partículas del material injertado existía formación ósea, confirmando las propiedades osteoconductivas del material injertado en acuerdo también con otros autores que señalan esta condición (Ruhaimi 2001, Jensen et al., 2006); no fue posible observar en estos periodos reabsorción o remodelación de las particular de la matriz bovina injertada.
La imagen radiográfica utilizada como apoyo al estudio histológico mostró diferencias en los resultados; existió una clara relación entre la imagen radiográfica e histológica en el hueso autógeno injertado, mostrando en la imagen radiográfica áreas radiopacas en relación a la fase de reparación ósea histológica. Sin embargo, en los defectos con material bovino se observaron también áreas radiopacas dentro del defecto lo cual podría señalar la presencia de hueso; sin embargo, lo observado en la imagen histológica era el propio material bovino injertado presente en las diferentes etapas del estudio y no hueso neoformado como se pudo haber pensado en el análisis radiográfico. Ferrús-Torres et al., 2009 señalaron que las tomografías computarizadas y las radiografías presentarían semejantes condiciones para realizar los seguimientos de procedimientos quirúrgicos, aunque Nolff et al. (2010) demostraron que las tomografías computarizadas no eran elementos de valor para evaluar la formación ósea en sitios injertados; nuestros resultados concuerdan con los de Nolff et al., en términos de señalar que no se observó una relación entre formación ósea y la imagen radiográfica en sitios injertados con biomaterial.

Finalmente podemos concluir que el vehículo de carboxi-metil-celulosa no fue eficiente en la formación ósea al ser comparado con vehículos de suero fisiológico.

DUQUE NETTO, H.; OLATE, S.; KLÜPPEL, L.; MIRANDA CHAVES, M. G. A.; SALGADO, I. O.; VÁSQUEZ, B. \& ALBERGARIA-BARBOSA, J. Bone regeneration in critical defect treated with inorganic bovine bone matrix with two different carriers. Int. J. Morphol., 31(2):367-372, 2013.

SUMMARY: In bone reconstruction has been used different bioamterials; bovine bone matrix has been studied and nowadays new research analyses the capacity for bone regeneration. The aim of this research was to evaluate the bone reparation in critical defects on skull of dog filled with inorganic bovine bone matrix carried by carboxi-methyl-celulose. Was design an experimental research with 6 dog; on parietal bone was realized a $8 \mathrm{~mm}$ diameter defect with a trephine and the defect was filled by particle autogenous bone (group I), inorganic bovine bone matrix carried with saline solution $(0,9 \%)$ (group II) and inorganic bovine bone matrix carried with carboxi-methyl-celulose. Was realized the sacrifice of animals in a third and sixth week and was performed the radiographic image and the histological study with hemaotoxilin-eusin in a routine technique. Non infection was observed in any of sample. In the autogenous bone graft was observed an adequately bone formation in the third and sixth week analyses and was related to radiographic image; for group II was observed some bone formation and presence of bovine bone 
particles and for group III was observed inflammatory cells for two period of analyses with a low level of bone formation. The radiographic analyses show sign of bone formation but histological analyses show only permanence of bovine bone particles confounding the radiograph results. Is conclude that bovine bone inorganic matrix in COMPATIBLE con bone tissue and can contributed to bone formation although the use of carboxi-methylcelulose can be an obstacle for bone regeneration.

\section{KEY WORDS: bone, bovine bone, osseous graft.}

\section{REFERENCIA}

Basle, M. F.; Grizon, F.; Pascaretti, C.; Lesourd, M. \& Chappard, D. Shape and orientation of osteoblast-like cells (saos 2) are influenced by collagen fibers in xenogenic bone biomaterial. $J$. Biomed. Mat. Res., 40:350-7, 1998.

Boyne, P.J.; Lilly, L.C.; Marx, R.E.; Moy, P.K.; Nevins, M.; Spagnoli, D.B. et al. De novo bone induction by recombinant human bone morphogenetic protein-2 (rhBMP- 2) in maxillary sinus floor augmentation. J. Oral. Maxillofac. Surg., 63:1693-707, 2005.

Chaves-Netto, H.D.M.; Olate, S.; Chaves, M.G.A.M. \& Barbosa, A.J.R.; Mazzonetto, R. Análisis histológico del proceso de reparación en defectos óseos. Reconocimiento de defectos críticos. Int. J. Morphol., 27:1121-7, 2009.

Degidi, M.; Artese, L.; Rubini, C.; Perrotti, V.; Iezzi, G. \& Piattelli, A. Microvessel density and vascular endothelial growth factor expression in sinus augmentation using Bio-Oss ${ }^{\circledR}$. Oral Disease, 12:469-75, 2005.

Ferrús-Torres, E.; Gargallo-Albiol, J.; Berini-Aytés, L. \& Gay Escoda, C. Diagnostic predictability of digital and conventional panoramic radiographs. Int. J. Oral Maxillofac. Surg., 38:11847, 2009

Grageda, E. Platelet-rich plasma and bone graft materials: a review and standardized research protocol. Implant Dent., 13:301-9, 2004.

Haas, R.; Haidvogl, D.; Donath, K. \& Watzek, G. Freeze-dried homogeneous and heterogeneous bone for sinus augmentation in sheep: Part I - Histological findings. Clin. Oral Impl. Res., 13:396-404, 2002.

Hollinger, J.O. \& Kleinschmidt, J.C. The critical size defect as an experimental model to test bone repair materials. J. Craniofac. Surg. 1:60-8, 1990.

Jensen, S.S.; Broggini, N.; Hjorting-Hansen, E.; Schenk, R. \& Buser, D. Bone healing and graft resorption of autograft, anorganic bovine bone $\beta$-tricalcium phosphate. A histologic and histomorphometric study in the mandible of minipigs. Clin. Oral Impl. Res., 17:237-43, 2006.
Moore, W. R.; Graves, S. E. \& Bain, G. I. Synthetic bone substitutes. A. N. Z. J. Surg., 71: 354-61, 2001

Nolff, M.C.; Kokemueller, H.; Hauschild, G.; Fehr, M.; Bormann, K.H.; Spalthoff, S.; et. al. Comparison of computed tomography and microradiography for graft evaluation after reconstruction of critical size bone defects using beta-tricalcium phosphate. J. Craniomaxillofac. Surg., 38:38-46, 2010

Olate, S.; Oliveira, G.R.; Jaimes, M. \& Albergaria-Barbosa, J.R. Recuperación ósea en procedimientos de reconstrucción y colocación de implantes. Int. J. Morphol., 25: 649-57, 2007.

Pallesen, L.; Schou, S.; Aaboe, M.; Hjorting-Hansen, E.; Nattestad, A. \& Melsen, F. Influence of particle size of autogenous bone grafts on the early stages of bone regeneration: a histologic and stereologic study in rabbit calvarium. Int. J. Oral Maxillofac. Implants, 17:498-506, 2002.

Ruhaimi, K.A. Bone graft substitutes: A comparative qualitative histologic review of current osteoconductive grafting materials. Int. J. Oral Maxillofac. Implants, 16:105-14, 2001.

Santa-Comba, A.; Pereira, A.; Lemos, R.; Santos, D.; Amarante, J.; Pinto,M.; Tavares,P. \& Bahia,F. Evaluation of carboxymethylcellulose, hydroxypropylmethylcellulose, and aluminum hydroxide as potential carriers for rhBMP-2. Journal of Biomedical Materials Research, 55(3):396-400, 2001.

Serra e Silva, F.; Albergaria-Barbosa, J.R. \& Mazzonetto, R. Clinical evaluation of association of bovine organic osseous matrix and bovine bone morphogenetic protein versus autogenous bone graft in sinus floor augmentation. J. Oral Maxillofac. Surg., 64: 931-5, 2006.

Vance, G.S.; Greenwell, H.; Miller, R.L.; Hill, M.; Johnston, H. \& Scheetz, J.P. Comparison of an allograft in an experimental putty carrier and a bovine-derived xenograft used in ridge preservation: a clinical and histologic study in humans. Int. J. Oral Maxillofac. Implants., 19:491-7, 2004.

Dirección para correspondencia:

Prof. Dr. Sergio Olate Morales

Facultad de Odontología

Universidad de La Frontera

Claro Solar $115,4^{\circ}$ Piso, Oficina 20

Temuco

CHILE

Email: sergio.olate@ufrontera.cl

Recibido : 16-01-2013

Aceptado: 22-03-2013 